\title{
On the Incorrect Derivation and Use of In-situ Retardation Factors from Natural Isotope Profiles
}

\author{
By I. G. McKinley ${ }^{1}$ and W. R. Alexander ${ }^{2}$ \\ ${ }^{1}$ Nagra (National Cooperative for the Disposal of Radioactive Waste), Wettingen, Switzerland \\ 2 GGWW (Rock Water Interaction Group), University of Berne, Berne, Switzerland
}

(Received September 15, 1995; accepted February 6, 1996)

\section{Sorption / Retardation factors / \\ Natural series radionuclides / Natural analogues / Kd / Modelling}

\begin{abstract}
The characterization of the retardation of radionuclides during their transport by deep groundwaters is complex, time-consuming and, in many cases, inherently limited by technical problems. The stated goal of many natural analogue studies is thus the direct quantification of solute retardation in-situ in relevant geological environments. This paper reviews the claims for a methodology for determining in-situ retardation factors from a mathematical deconvolution of multiple radioisotope measurements of natural decay series elements. It is concluded that the assumptions involved in development of the mathematical approach are geochemically inconsistent and inapplicable to most geological systems of interest. Misapplications of this model are identified and the dangers of accepting its nonsensical output are discussed.
\end{abstract}

\section{Introduction}

The term "modelling" is loosely applied to many different procedures. For example, truely predictive models may be very different from those which merely simulate observations using curve fitting procedures. Similarly, within the radioactive waste management field, it is important to distinguish between "realistic" models intended to make predictions which are as accurate as possible and "conservative" models which are known to be inaccurate but are intended to take uncertainties into account by overpredicting consequences.

In large multi-disciplinary projects, considerable opportunities for confusion arise. This has been particularly the case in the natural analogue field, where observations of natural systems are used to support, test or provide input for repository performance assessment models. Here similar (or identical) parameter names are used by geochemical and performance assessment groups for quantifying significantly different processes. In many cases, when communication between these two groups is not ensured, confusion results in conclusions being drawn from analogue studies which are incorrect and potentially dangerous if used directly in safety assessment.

In previous papers $[1,2,3]$ we have examined the way in which rather simplistic assessment of the pro- cesses causing partitioning of solute between rock and an aqueous phase has resulted in incorrect claims for the applicability of "in-situ $K d$ " data to performance assessment. Recently, a more complex approach to interpretation of natural series radionuclide data has been claimed to yield a wide range of solute/rock interaction parameters [4] which are relevant for performance assessment [5]. This paper examines the applicability of the simulation model itself and further considers the extent to which any data derived can be extrapolated to other systems/type of model - particularly within the context of radioactive waste management.

\section{Distribution of natural decay series radionuclides in groundwater and rocks}

The natural decay series results from the radioactive decay of the long-lived nuclides U-238, U-235 and Th232 via a chain of shorter lived daughters. In an undisturbed rock which is sufficiently old for the daughters to have grown into secular equilibrium, the activity of a parent radionuclide is identical to that of all its daughters. In an open system, the differing chemistry of the daughters and the physico-chemical effects of radioactive decay tend to cause preferential loss of particular nuclides so that parent/daughter activity ratios can deviate markedly from unity [6]. In a system of groundwater in contact with rock, the resultant isotope ratios in solution are further complicated by processes which can transfer dissolved species back to the solid phase (e.g. sorption and precipitation, discussed further below). If the groundwater flows through a series of rocks, the isotope ratios at any particular location will reflect its history of interactions with the rock.

Despite the evident complexity of the system, there have been many attempts to try to interpret isotope ratios in rock and groundwater quantitatively in terms of the individual processes involved. One approach is to try to separate components of the system which have been affected by different processes (e.g. distinguishing between sorbed, co-precipitated and matrix components of the solids) but this is experimentally very problematic and the results produced are often highly questionable [2]. Alternatively, complex mathematical models can be constructed in an attempt to 
deconvolute multiple isotope measurements on a single sample $[4,5]$.

\section{The model of $\mathrm{Ku}$}

Within a review of models of natural series disequilibrium during rock/water interaction, Ku et al. [4] develop a calculational approach to quantify the retardation factor, rate constants for adsorption, desorption and precipitation and mean residence time of an element in solution based on measurements of solution activities of isotopes of $\mathrm{U}, \mathrm{Th}, \mathrm{Ra}, \mathrm{Rn}, \mathrm{Pb}$ and $\mathrm{Ac}$. In order to develop this model, very many assumptions (both explicit and tacit) must be made. For example, the model focuses on "one-dimensional porewater flow and constant Darcy velocity" - which may be appropriate for aquifer studies but not for many geological systems of interest.

Some of the most fundamental assumptions apply to the representation of sorption and precipitation. Radionuclides are assumed to reside in 3 pools: solid, sorbed and dissolved (i.e. the presence of colloids is excluded). The description of the "solid phase" by $\mathrm{Ku}^{1}$ is somewhat confusing as it is stated that "it is assumed that dissolution and precipitation of solids occur irreversibly, that the adsorbed pool resides in a surface layer which provides the sites for reversible ion exchange" and that "it is also envisaged that upon attack by weathering solutions, rocks undergo incongruent dissolution, leading to partial release of their elemental constituents and to the formation of a surface layer referred to above". The model also "places the solid-solution interface at the boundary between the surface layer and the solid phase". The separation of dissolving and precipitating components is clear mathematically, but it is implied that the precipitates are effectively inert, i.e. the in-situ production of daughter from sorbed parent is taken into account but not that from precipitated parent, which appears extremely unrealistic.

Precipitation is represented by a first order rate constant $(k p)$, i.e. rate of precipitation loss $=k p C$ where $C$ is concentration of a dissolved radionuclide. This is fine as a model fit parameter, but has nothing to do with precipitation as considered in geochemistry where, for precipitation of a pure mineral phase, the rate might be $k p\left(C_{\mathrm{E}}-C_{\text {sat }}\right)$ where $C_{\mathrm{E}}$ is the total elemental concentration (summed up over all isotopes of an element) and $C_{\text {sat }}$ is the solubility limit of the solid considered. In reality, except for in the vicinity of ore bodies, the formation of pure minerals would not be expected and co-precipitation (with, for example, iron oxyhydroxide) would be more likely. In practice, it is likely to be extremely difficult to distinguish sorption from co-precipitation but there are fundamental differences between these processes which are critical from a modelling point of view:

\footnotetext{
"as pointed out by anonymous reviewer, this is not a "phase" in the thermodynamic sense and its use by $\mathrm{Ku}$ is rather vaguely defined.
}

a) Sorption is related to the availability of suitable surfaces (external and internal) and the concentration in the sorbate is a function of the concentration in solution (in reality, the function may be complex but can often be approximated by a partition coefficient or an isotherm).

b) Precipitation occurs only when a solubility limit for a particular solid has been exceeded and the total inventory of a precipitated phase is dependent on the rate and duration of precipitation (for co-precipitation, the extent of incorporation of the nuclide of interest is also a key factor) and not in any way on the instantaneous solution concentration.

By taking the simplest possible representation of sorption (completely reversible, fast sorption where the ratio of the "equilibrium" concentration of nuclide in the sorbate to the concentration in solution is a constant), a retardation factor $(R f)$ can be defined which is equivalent to the ratio of the flow rate of fluid to that of contained nuclide if sorption is the only process causing retention. $\mathrm{Ku}$ takes this concept one stage further by including precipitation in a novel type of retardation factor:

$$
R f^{*}=R f+\frac{k p}{\lambda}
$$

$R f$ and $k p$ are as previously defined and $\lambda$ being the decay constant of the nuclide of interest. The claim of the significance of $R f^{*}$ as a retardation factor including sorption and precipitation is particularly confusing. $R f^{*}$ here is completely dependent on all the assumptions above plus irreversible precipitation being a function of the total nuclide concentration in solution. At the same time, "irreversible dissolution" (Ku's terminology) is assumed to be only dependent on the total concentration of nuclide in the matrix rock and not in any way on the solution concentration. The particular problems with this parameter are clear if the case of a stable element is considered; here $\lambda=0$ and thus $R f^{*}=\infty-$ which is nonsensical as the modelling of the sorption and precipitation processes are not related to the decay process in any way.

The fundamental problem with this formulation is clear also for radionuclides, as it implies $R f^{*}$ is proportional to half-life for $k p / \lambda \gg R f$. This would mean that isotopes could readily be separated chromatographically if their half-lives differed significantly (clearly nonsensical and evident from, for example, the observed constancy of the U-235/U-238 ratio in nature). The discussion of the relevance of $R f^{*}$ is very obtuse, but terming it a "retardation factor" is certainly very confusing. Further confusion is also evident when the $R f^{*}$ of very short-lived nuclides is considered in detail.

In the illustration of the application of the model to sets of measured data, $\mathrm{Ku}$ et al. present no further discussion of whether the major assumptions involved are reasonable in the system studied (e.g. the assumption that the sorption distribution coefficient is independent of concentration - experimentally demon- 
strated not to be the case in many circumstances) and, indeed, further simplifications are introduced in order to solve the complex sets of equations derived (e.g. that the fractional release rate from the rock matrix during weathering is the same for all isotopes - which would certainly not be expected for Th-232, Th-234 and Th-230).

Although $\mathrm{Ku}$ states "... the general approach ... of determining the in-situ adsorption/desorption rate constants and retardation factors should find application in developing site-specific models of the transport of radioactive and stable elemental waste through watersaturated media" [4, p651], it is obvious that the fit parameters used in the model are not equivalent to similarly named parameters used by solute transport modellers. Further, the extent of applicability of the model is likely to be extremely limited - restricted to the rare systems in which all of the sequence of assumptions used to derive practicable mathematical solutions are reasonable.

\section{Application of Ku's model}

One of the most dangerous things about models which contain large numbers of fitting parameters is that they can generally be forced to simulate any particular set of observational data. The key question is whether the model-fitting exercise is meaningful or not. Two approaches are useful to screen such applications checking whether the key assumptions of the model are applicable to the system studied and evaluation of the consistency of output parameter values.

As far as consistency checks on derived parameters are concerned, it is first essential that all values which relate directly to physical processes are physically reasonable - e.g. derived surface areas must be positive. In such a complex model, the production of any unreasonable value calls the entire model into question. Particular values must also be sensible in context; thus sorption parameters derived independently for various long-lived isotopes of a particular element should be identical (within the inherent uncertainty involved).

The dangers involved in utilization of such a complex model without a careful evaluation of its applicability are illustrated in a study in which "the specific aim was to use a simple model to determine the rock/ water exchange timescales and retardation factors for uranium and thorium isotopes in a Triassic sandstone aquifer ... chosen because it is a reasonable analogue to the far field of a number of potential sites for radioactive waste disposal" [5, p423].

Even from a superficial review of the description of the system examined, it is clear that the entire basis of Ku's model - assuming $1 \mathrm{D}$ flow through a homogeneous porous media - is unlikely to be applicable to a system in which "both fissure and intergranular flow occur". At the very least, scoping calculations would be needed to determine the consequences of slow transport from the intergranular matrix into the fissures which are likely to dominate the inflow into the boreholes sampled.

The sampling procedures also seem to have paid little heed to the assumptions involved in the model to be applied. Given that the model considers a simple steady-state sub-system, it seems inappropriate to utilize samples from uncased boreholes so that "to some extent, all samples are mixtures of water from different horizons". Equally seriously, the water samples were filtered using only $1 \mu \mathrm{m}$ filter cartridges and hence inevitably contain a colloidal component which is completely uncharacterized. Given that $\mathrm{Th}$, in particular, is often present in waters filtered to $<450 \mathrm{~nm}$ predominantly in a colloidal form $(\sim 60-90 \% ;[7])$ and readily associates with such colloids in sorption experiments [8], this calls into question the validity of all subsequent analysis with a model based on the assumption that no colloids are present.

In addition to the groundwater samples, some rock samples were also measured by Ivanovich et al. [5]. A sequential leaching procedure was used to determine activities in a "surface layer" (e.g. Table 1). It is not evident from the paper exactly how these "surface layer" data are interpreted, but inspection shows clearly that the massive isotopic ratio discrepancies between water and rock samples clearly indicate that these values cannot possibly be produced by sorption processes alone. In fact, the difference between total and surface rock U-234/U-238 ratios indicate that the residual rock must have a ratio $>1.24$ for the $50 \mathrm{~m}$ sample and $<0.90$ for the $200 \mathrm{~m}$ sample, implying that the materials involved and/or the processes to which they have been subjected are very different for these 2 samples (as the U-234/U-238 in water is $\gg 1$ for both cases).

The clearest demonstration of problems with the data interpretation is provided by the output parameters derived. Although retardation factors calculated separately for U-234 and U-238 are claimed to be "quite similar" - they differ by factors of up to 350 ! As noted above, there is no credible physical justification for significant isotopic fractionation due to sorption (or even precipitation, if it is also rolled into a "retardation factor"). Less demonstrably wrong, but certainly a warning signal, are the incredibly short "time in solution before irreversible sorption" values quoted for Th $(\sim 0.3-3$ seconds). Although the nomenclature is very confusing, such times seem extremely short and differ by many orders of magnitude from times $\sim$ days observed in laboratory studies for Th sorption on sediments [8].

In conclusion, therefore, the claim of the paper that "Results obtained using the protocol described may be compared with similar data derived from small scale, laboratory experiments over limited times, which are used in repository performance assessment modelling to improve confidence in such modelling" [5, p431] is completely unjustified. It is clear that the model fit parameters derived have nothing to contribute to solute 
Table 1. $U+$ Th activity ratios in rock and water samples reported in [5]

\begin{tabular}{lccc}
\hline Sample & U-234/U-238 & Th-230/U-234 & Th-238/Th-232 \\
\hline Chat Moss 50 m & & & \\
- Water & $7.27( \pm 0.14)$ & $0.001( \pm 0.0003)$ & $7.3( \pm 4.0)$ \\
- Rock (total) & $1.24( \pm 0.06)$ & $1.13(0.06)$ & $1.01( \pm 0.05)$ \\
- Rock (surface) & $1.08( \pm 0.54)$ & $0.62( \pm 0.21)$ & $1.92( \pm 0.18)$ \\
& & & \\
Chat Moss 200 m & & & \\
- Water & $13.77( \pm 0.43)$ & 0 & $( \pm 10)$ \\
- Rock (total) & $0.90( \pm 0.04)$ & $0.93( \pm 0.08)$ & $0.96( \pm 0.09)$ \\
- Rock (surface) & $2.21( \pm 0.54)$ & $0.73( \pm 0.16)$ & $2.00(0.40)$ \\
\hline
\end{tabular}

transport modelling and, due to the fundamental limitations of the model/dataset used, have probably no physical meaning of any kind.

\section{Perspective and conclusions}

Development of models in order to interpret observations in a quantitative manner is a keystone of science and, inevitably, models of natural geological systems start by introducing a number of simplifying assumptions in order to make the analysis tractable. It is important, however, to ensure that the quest for elegant equations which can be readily solved does not result in a product which is inapplicable to the system of interest.

This general requirement is critical if it is claimed that data are produced which are relevant for the assessment of radioactive (or toxic) waste management options. Studies of the type criticised in this paper cause three types of problems:

a) They divert funds/resources from more relevant work; given that laboratory studies are extremely expensive and time-consuming, the claim for production of good, cheap data from "site-specific" studies can be attractive if the limitations of the resultant output are not noted.

b) They call into question the effectiveness of the scientific peer-review process; the technical credibility of repository safety assessments is founded on the publication of supporting research in the open literature with the implicit assumption of quality assurance by the technical community.

c) They create the risk that results produced could be taken at face value and used in a performance assessment; although this is unlikely in programmes with established QA/QC procedures, such a risk is significant for developing countries with low budgets and significant waste disposal problems.

The last point is especially relevant. One thing clearly shown by past experience is that, despite the relatively high costs of waste disposal, the costs of site remediation can be orders of magnitude higher if the disposal option adopted is inappropriate!

The conclusions from this study may be self-evident, but seem to need emphasis: a) Mathematical models of natural systems are inevitably gross simplifications which must be carefully reviewed to ensure that the quest for ease of solution does not clash with common sense.

b) The applicability of a model to any particular system must be checked in advance and, at the very least, the effect of major discrepancies between model assumptions and field observations assessed by scoping calculations.

c) Sample selection should also take the fundamentals of the model into account and, for radioactive waste management applications, the quality of the sample should be ensured in an appropriate manner.

d) The parameters derived from the model should be subjected to "reality checks". Any values which are clearly physically unreasonable call the entire basis of the model/database used into question.

e) Output parameters should be compared for consistency with equivalent values derived from laboratory studies; massive discrepancies should be identified and discussed.

Although this paper has focused on a particular study area, similar problems certainly exist elsewhere. The volume of literature being produced probably makes failures of QA during peer review inevitable, hence it is strongly recommended that periodic critical reviews (not bibliographic "stamp collection") are carried out for the different methods used to provide natural systems data for performance assessment.

\section{Acknowledgments}

The authors are grateful to Nagra (National Cooperative for the Disposal of Radioactive Waste) for supporting this work and to colleagues in the natural analogues and performance assessment communities who have provided feedback on our previous papers in this topic.

\section{References}

1. McKinley, I. G., Alexander, W. R.: J. Environ. Radioactivity 15, 19-34 (1992). 
2. McKinley, I. G., Alexander, W. R.: J. Contaminant Hydrology 13, 249-259 (cf. also Comments and Response 261-275) (1993).

3. Alexander, W. R., McKinley, I. G.: Eclogae geol. Helv. 87, 321-324 (1994)

4. Ku, T.-L., Luo, S., Leslie, B. W., Hammond, D. E.: Decayseries disequilibria applied to the study of rock-water Uranium-Series Disequilibrium, 2 nd edn. (eds. M. Ivanovich, R. S. Harmon), Clarendon Press, Oxford (1992).
5. Ivanovich, M., Tellam, J. H., Longworth, G., Monaghan, J. J., Radiochim. Acta 58/59, 423-432 (1992).

6. Osmond, J. K., Ivanovich, M., Uranium-series mobilisation and surface hydrology, in: Uranium-Series Disequilibrium, 2nd edn. (eds. M. Ivanovich, R. S. Harmon), Clarendon Press, Oxford (1992).

7. Miekeley, N., Coutinho de Jesus, H., Porto da Silveira, C. L., Degueldre, C.: J. Geochem. Explor, 45, 409-437 (1992).

8. Lieser, K. H., Hill, R.: Radiochim. Acta 56, 141-151 (1992). 
